\title{
Nephrotic-range proteinuria in an infant with thin basement membrane nephropathy
}

\author{
Shingo Ishimori $\cdot$ Hiroshi Kaito $\cdot$ Shigeo Hara $\cdot$ \\ Koichi Nakanishi $\cdot$ Norishige Yoshikawa \\ Kazumoto Iijima
}

Received: 23 October 2012/Accepted: 7 January 2013/Published online: 16 February 2013

(C) Japanese Society of Nephrology 2013

\begin{abstract}
Thin basement membrane nephropathy (TBMN) with heterozygous COLAA3/COL4A4 mutations is considered to be a cause of benign familial hematuria. The disease has been believed to have excellent prognosis and TBMN in early childhood is rarely associated with nephrotic-range proteinuria. Furthermore, the presence of proteinuria in patients with TBMN is associated with autosomal-dominant Alport syndrome, which has poorer prognosis in later life. We present an infant case of nephrotic-range proteinuria associated with TBMN caused by heterozygous COL4A4 mutation. A previously healthy 3-year-old boy developed microhematuria and nephroticrange proteinuria. Renal pathology simply revealed thinning of the glomerular basement membrane (GBM) and mutational analysis revealed a novel heterozygous mutation in COLAA4. He was treated with lisinopril for 1.5 years, which resolved his proteinuria and hematuria. At the most recent follow-up at 6.5 years of age, urinalysis and kidney function were completely normal, without requiring medication. However, transient but repeated moderate to nephrotic-range proteinuria and microscopic hematuria occurred in association with other illnesses. This case highlights the spectrum of phenotypes that may be
\end{abstract}

S. Ishimori $\cdot$ H. Kaito $(\bowtie) \cdot$ K. Iijima

Department of Pediatrics, Kobe University Graduate School of

Medicine, 7-5-1 Kusunoki-cho, Chuo, Kobe,

Hyōgo 6500017, Japan

e-mail: hkaitoh@med.kobe-u.ac.jp

S. Hara

Division of Diagnostic Pathology, Department of Pathology,

Kobe University Graduate School of Medicine, Kobe, Japan

K. Nakanishi · N. Yoshikawa

Department of Pediatrics, Wakayama Medical University,

Wakayama, Japan apparent in an infant with TBMN. Thinning of the GBM can cause transient nephrotic-range proteinuria, particularly in the early stages of TBMN.

Keywords Collagen type IV nephropathy · Heterozygous COL4A4 mutation - Proteinuria . Thin basement membrane nephropathy

\section{Background}

Thin basement membrane nephropathy (TBMN) is a phenotypic variant of autosomal-dominant collagen type IV nephropathy, which is caused by heterozygous mutations of the type IV collagen genes, COLAA3 and COLAA4. TBMN with heterozygous $C O L 4 A 3 / C O L 4 A 4$ mutations is considered to be a cause of benign familial hematuria and TBMN in early childhood is rarely associated with nephrotic-range proteinuria. Here, we report a 3-year-old boy with TBMN with a heterozygous COLAA4 mutation who experienced transient but repeated varying degrees of proteinuria.

\section{Case report}

A previously healthy 3-year-old boy with no family history of renal disease was referred to our hospital because of hematuria and proteinuria at his 3-year checkup. He had no clinical symptoms, and all of his vital signs and physical examinations were normal. His urinary protein/creatinine ratio (u-P/Cr) was $3.2 \mathrm{~g} / \mathrm{gCr}$ and microhematuria was detected at the initial visit. His blood urea nitrogen, serum creatinine, and serum albumin levels were 12, 0.26, and $2.9 \mathrm{~g} / \mathrm{dL}$, respectively. Serum complement levels and renal ultrasound findings were normal. A renal biopsy was 
performed 30 days after detecting the urinary abnormality, because nephrotic-range proteinuria persisted. The glomeruli were almost normal under a light microscope, except for some proliferation of mesangial cells. Immunohistochemical analyses, including type IV collagen $\alpha 5$, were completely normal. The width of the glomerular basement membrane (GBM) was $150 \mathrm{~nm}$ under an electron microscope, which indicated global thinning of the GBM [1]. There was no evidence of basket-weave or split lesions (Fig. 1). Foot process fusion was partly apparent, but these areas covered a mere $30 \%$ of the visible range and it was not compatible with primary minimal change nephrotic syndrome. The direct sequencing of COLAA4 with genomic DNA from peripheral blood lymphocytes revealed that the patient and his mother had a heterozygous mutation of $\mathrm{c}$. $827 \mathrm{G}>\mathrm{C}$ (p. G276A) in exon 14, but his father did not (Fig. 2). We confirmed that a hundred healthy controls did not have the same mutation and that this is a diseasecausing mutation. Based on these results, we diagnosed the patient with TBMN with a heterozygous COLAA4 mutation. Therefore, treatment with an angiotensin-converting enzyme inhibitor (lisinopril $0.4 \mathrm{mg} / \mathrm{kg} / \mathrm{day}$ ) was started. His urinary abnormality and serum albumin level gradually improved, and lisinopril was discontinued 1.5 years after starting treatment. In 3.5 years after the initial presentation, i.e., at 6.5 years of age, his urinalysis and kidney function were normal, without requiring medication. However, the patient did develop transient episodes of moderate to nephrotic-range proteinuria and microscopic hematuria that lasted for approximately 1 month per episode. These events occurred in association with other illnesses, including pharyngitis and acute gastroenteritis.

\section{Discussion}

It is quite rare for infants with TBMN, such as this case, to develop nephrotic-range proteinuria. Pierides et al. [2] reported that TBMN associated with heterozygous COL4A3/COLAA4 mutations only resulted in microscopic hematuria or urinalysis that was negative in patients aged $<30$ years. In patients with Alport syndrome, a severe form of collagen type IV nephropathy, the initial renal manifestation is asymptomatic persistent microscopic hematuria, similar to that in TBMN. Proteinuria gradually develops over time in Alport syndrome after the development of longitudinal splits and lamellations of the GBM lamina densa. However, in our case, interestingly, we found no changes in the GBM other than diffuse thinning. Other researchers have reported that adults with TBMN may have moderate to nephrotic-range proteinuria of unknown etiology, with no changes in the GBM other than thinning $[3,4]$. Our case showed that thinning of the GBM itself is associated with nephrotic-range proteinuria, even in the early stages of TBMN.

Type IV collagen, a component of GBM, is constructed of three $\alpha$ chains to form a triple helical molecule, and mutations in COLAA4 produce a posttranslational defect that prevents the correct structure of the collagen type IV network. Our patient has a mutation in the collagenous domain of COLAA4, which probably interferes with the proper formation and structure of the collagen triple helix. Type IV collagen nephropathy is clinically heterogenous [5], and it seems probable enough that our patient revealed nephrotic-range proteinuria as a consequence of thinning of the GBM associated with COL4A4 mutation.
Fig. 1 Electron microscopy of a kidney biopsy shows thinning of the glomerular basement membrane (GBM). a The width of the GBM was $150 \mathrm{~nm}$ at 10,000-fold magnification. b The area of foot process fusion covered about $30 \%$ of the visible range at 3,000-fold magnification. Basket-weave and split lesions were not shown. c Light microscopy showed no signs of focal segmental glomerulosclerosis (FSGS) [periodic acid-Schiff (PAS) staining with $400 \times$ magnification].

d Immunohistochemical staining of type IV collagen $\alpha 5$ were completely normal

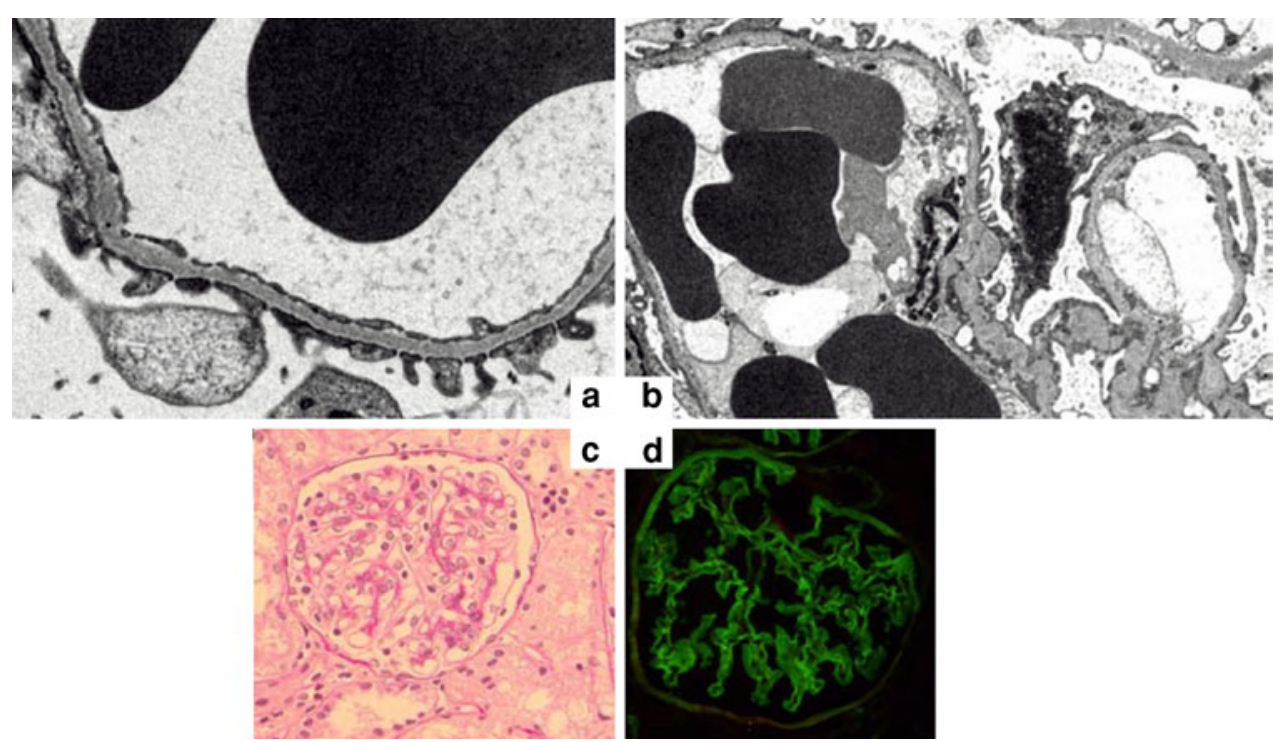


Fig. 2 DNA chromatograms in forward frames of the patient, his parents, and a normal control. A heterozygous transition $(\mathrm{G}>\mathrm{C})$ at nucleotide 827 located in exon 14 of the patient resulted in a glycine to alanine substitution at amino acid 276 (c. $827 \mathrm{G}>\mathrm{C}$, p.G276A)
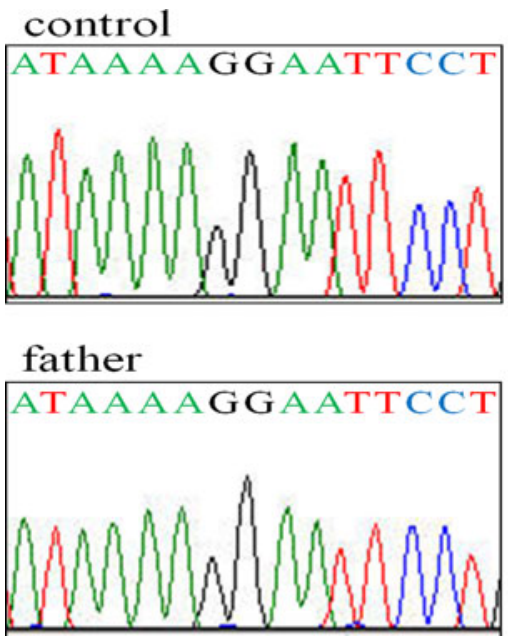

patient

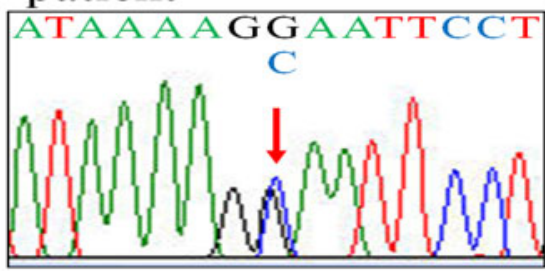

mother

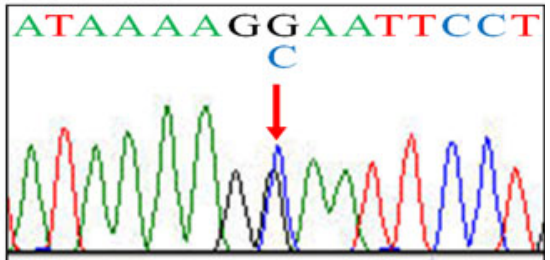

Our case is of special interest in that varying degrees of proteinuria developed transiently and repeatedly. The patient's mother had negative urine findings with the same mutation of COLAA4. The different phenotype of the same mutation may be explained by the involvement of chemical mediators or other structural proteins, such as the glomerular podocyte, which may interact with type IV collagen [6, 7]. Our patient's phenomena that he showed mild to nephrotic-range proteinuria only in various episodes of acute infection may suggest the involvement of chemical mediators such as cytokine or chemokine. Although it may remain possible that the patient has nephrotic syndrome associated with TBMN, we have evaluated that his unique clinical course could sufficiently reduce this possibility.

About $20 \%$ of patients with TBMN show progressive renal impairment [8]. TBMN associated with heterozygous COL4A3/COLAA4 mutations was reported to show a causal relationship with the development of focal segmental glomerulosclerosis (FSGS) and renal failure [4, 9, 10]. TBMN itself may be a primary factor leading to chronic renal insufficiency and FSGS may be a consequence of proteinuria, though our patients had no sign of FSGS under light and electron microscopy at the time of biopsy.

In conclusion, this case highlights the unique phenotype of TBMN in an infant. Thinning of the GBM itself can cause transient nephrotic-range proteinuria, even in the early stages of TBMN. It is possible that the late development of chronic kidney insufficiency may be related to these episodes. Long-term observation is essential for patients with TBMN.

Conflict of interest None.

\section{References}

1. Vogler C, McAdams AJ, Homan SM. Glomerular basement membrane and lamina densa in infants and children: an ultrastructural evaluation. Pediatr Pathol. 1987;7:527-34.
2. Pierides A, Voskarides K, Athanasiou Y, Ioannou K, Damianou L, Arsali M, et al. Clinico-pathological correlations in 127 patients in 11 large pedigrees, segregating one of three heterozygous mutations in the COL4A3/COL4A4 genes associated with familial haematuria and significant late progression to proteinuria and chronic kidney disease from focal segmental glomerulosclerosis. Nephrol Dial Transplant. 2009;24:2721-9.

3. Dische FE, Weston MJ, Parsons V. Abnormally thin glomerular basement membranes associated with hematuria, proteinuria or renal failure in adults. Am J Nephrol. 1985;5:103-9.

4. Nieuwhof CM, de Heer F, de Leeuw P, van Breda Vriesman PJ. Thin GBM nephropathy: premature glomerular obsolescence is associated with hypertension and late onset renal failure. Kidney Int. 1997;51:1596-601.

5. Pescucci C, Mari F, Longo I, Vogiatzi P, Caselli R, Scala E, et al. Autosomal-dominant Alport syndrome: natural history of a disease due to COLAA3 or COLAA4 gene. Kidney Int. 2004;65: 1598-603.

6. Tonna S, Wang YY, Wilson D, Rigby L, Tabone T, Cotton R, et al. The R229Q mutation in NPHS2 may predispose to proteinuria in thin-basement-membrane nephropathy. Pediatr Nephrol. 2008;23:2201-7.

7. Voskarides K, Arsali M, Athanasiou Y, Elia A, Pierides A, Deltas C. Evidence that NPHS2-R229Q predisposes to proteinuria and renal failure in familial hematuria. Pediatr Nephrol. 2012;27:675-9.

8. Frascà GM, Onetti-Muda A, Mari F, Longo I, Scala E, Pescucci $\mathrm{C}$, et al. Thin glomerular basement membrane disease: clinical significance of a morphological diagnosis - a collaborative study of the Italian Renal Immunopathology Group. Nephrol Dial Transplant. 2005;20:545-51.

9. van Paassen P, van Breda Vriesman PJ, van Rie H, Tervaert JW. Signs and symptoms of thin basement membrane nephropathy: a prospective regional study on primary glomerular disease-The Limburg Renal Registry. Kidney Int. 2004;66:909-13.

10. Voskarides K, Damianou L, Neocleous V, Zouvani I, Christodoulidou S, Hadjiconstantinou V, et al. COLAA3/COLAA4 mutations producing focal segmental glomerulosclerosis and renal failure in thin basement membrane nephropathy. J Am Soc Nephrol. 2007;18:3004-16. 